\title{
Una taxonomía de términos clave en Educación Matemática ${ }^{1}$
}

\author{
A taxonomy of key terms in Mathematics Education
}

\section{A taxonomia de termos-chave em Educação Matemática}

Recibido: mayo de 2013

Aceptado: agosto de 2013
Pedro Gómez ${ }^{2}$

María C. Cañadas ${ }^{3}$

\section{Resumen}

La comunidad de investigadores, formadores y profesores de matemáticas ha producido una gran cantidad de literatura que se publica en libros, revistas, actas de congresos e Internet, entre otros. En la actualidad, no existe una taxonomía de términos clave que aborde sistemáticamente la clasificación de los documentos de esta disciplina. En este artículo, presentamos el procedimiento con el que construimos una taxonomía para Funes, un repositorio digital de documentos en Educación Matemática.

Palabras clave: Educación Matemática y otras disciplinas; Recursos informáticos; Software; Libros de texto.

\begin{abstract}
The community of researchers, educators and mathematics teachers has been a lot of literature published in books, magazines, conference proceedings and the Internet, among others. At present, there is a taxonomy of key terms systematically address the classification of the documents in this discipline. In this paper, we present the method with which we construct a taxonomy for Funes, a digital repository of documents in mathematics education.
\end{abstract}

Keywords: Metacognition, demonstration, geometry, problem solving, cognitive processes, processes of justification, teacher training.

\section{Resumo}

A comunidade de pesquisadores, educadores e professores de matemática tem sido um monte de literatura publicada em livros, revistas, anais de conferências e internet, entre outros. No momento, não há uma taxonomia de termos-chave abordar sistematicamente a classificação dos documentos nesta disciplina. Neste artigo, apresentamos o método com o qual construímos uma taxonomia para Funes, um repositório digital de documentos em educação matemática.

Palavras-chave: Educação Matemática e outras disciplinas recursos de computador, software, livros didáticos.

1 Artículo de Investigación

2 Universidad de los Andes, profesor. Contacto: argeifontes@gmail.com

3 Universidad de Granada, profesora. Contacto: mconsu@ugr.es 


\section{Introducción}

La Educación Matemática se ha consolidado como una disciplina en las últimas décadas. Aunque existen algunas aproximaciones a la clasificación, por medio de tesauros, de la documentación que se ha producido, no existe ninguna solución detallada y estructurada al problema de establecer un vocabulario controlado para la disciplina. Un vocabulario controlado es un sistema que permite la búsqueda y selección de contenidos a través de algún tipo de descripción lingüística. Su propósito principal es lograr una descripción consistente de los contenidos y facilitar su recuperación. Nuestro trabajo está basado en un estándar para la construcción, formato y gestión de vocabularios controlados (NISO, 2005). Según este estándar, el diseño y desarrollo de un vocabulario controlado sigue cuatro principios (p. 13): (a) eliminar la ambigüedad, (b) controlar los sinónimos, (c) establecer relaciones entre términos, cuando se considere apropiado, y (d) verificar y validar los términos. Al seguir estos principios, se buscan dos propósitos: precisión - encontrar todo lo que es posible encontrar- y relevancia - evitar resultados que no corresponden con los intereses-. En este trabajo, describimos el proceso que dio lugar a la construcción de la taxonomía de términos clave para Funes. ASOCOLME utiliza esta taxonomía para clasificar los documentos que se publican en las memorias de sus encuentros.

Funes es un repositorio digital de documentos en Educación Matemática (Gómez, Cañadas, Soler y Restrepo, 2009). Su objetivo es contribuir a la mejora de la enseñanza y aprendizaje de las matemáticas, poniendo a disposición de la comunidad de educadores matemáticos los documentos que no tienen restricción de derechos de autor y que pueden apoyar el trabajo de esta comunidad. Esta comunidad incluye, entre otros, investigadores en Educación Matemática, formadores de profesores de matemáticas y profesores de matemáticas de todos los niveles educativos. El contenido de Funes está disponible para todo el público en http://funes.uniandes.edu.co. Los documentos propuestos por sus autores para su publicación son revisados por un comité académico que verifica su legibilidad, completitud y coherencia.
En muchas ocasiones, el usuario busca información sobre un tema o problema sin conocer qué documentos tratan ese tema o dan respuesta a ese problema. En este caso, es necesario hacer una búsqueda por términos clave. El usuario espera un esquema de búsqueda que le permita identificar con rapidez y precisión los documentos más relevantes para sus intereses.

Teniendo en cuenta los principios de los vocabularios controlados mencionados anteriormente, nos propusimos establecer una taxonomía de términos clave para Funes. Para ello, identificamos tres tipos de términos clave que denominamos enfoque, nivel educativo y tema. El enfoque caracteriza el propósito y utilidad del documento-investigación, ensayo, innovación y actividad-, mientras que el nivel educativo identifica el tipo de formación de los sujetos a los que hace referencia el documento. El problema principal se centraba en los términos clave que denominamos "temas". Esta sería la taxonomía que debería permitir búsquedas precisas y relevantes a aquellos usuarios con un interés concreto. En nuestra primera aproximación a este problema, intentamos utilizar una taxonomía ya existente. Las taxonomías de la UNESCO (2010) y ERIC (2010), que son taxonomías generales reconocidas sobre educación, resultaron muy limitadas porque proporcionan un número reducido de términos clave en Educación Matemática. Decidimos entonces analizar MathEduc (FIZ Kalruhe, 2010), la base de datos de documentos especializada más conocida en Educación Matemática. Sin embargo, al analizar este tesauro, encontramos algunas dificultades que nos llevaron a construir nuestra propia taxonomía de términos clave. Nos impusimos, en todo caso, la condición de que todo término clave de MathEduc tuviera un término equivalente en Funes.

\section{Construcción de la taxonomía de Funes}

Buscamos que la taxonomía de Funes estuviese basada en un marco conceptual sólido y específico a la Educación Matemática y fuese eficiente para el registro y búsqueda de documentos (Pinto, 2008), al mismo tiempo que abordara los problemas de ambigüedad y sinonimia. Diferenciamos los términos clave que se referían a la Educación Matemática de aquellos que se referían a los contenidos matemáticos. 
Separamos estos últimos en términos clave de las matemáticas escolares y términos clave de las matemáticas superiores. Nos basamos en la taxonomía de TIMMS (Mullis, Martin, Ruddock, O’Sullivan, Arora y Eberber, 2005) y TEDS-M (Tatto, Schwille, Schmidt, Ingvarson y Beavis, 2006) para la construcción de estas dos taxonomías.

Para los términos clave de Educación Matemática, adoptamos un enfoque curricular (Rico, 1997). El currículo busca abordar cuatro cuestiones centrales (p. 381) sobre (a) el conocimiento a enseñar, (b) el aprendizaje, (c) los métodos de enseñanza y (d) la valoración de los aprendizajes realizados. Estas cuatro cuestiones básicas dan lugar a cuatro dimensiones conceptual, cognitiva, formativa y social $-\mathrm{y}$ a cinco niveles -fines, disciplinas, sistema educativo, planificación de profesores y planificación local一. Este enfoque nos condujo a las nueve categorías básicas de términos clave de Educación Matemática en Funes: (a) sistema educativo, (b) centro educativo, (c) aula, (d) alumno, (e) profesor, (f) aprendizaje, (g) enseñanza, (h) evaluación y (i) currículo. Por otro lado, incluimos una categoría para la relación entre la Educación Matemática y otras disciplinas, y otra categoría para los términos clave relacionados con la investigación y la innovación en Educación Matemática. También incluimos una categoría general para aquellos términos clave propios de la Educación Matemática que no tenían cabida clara en la estructura curricular.

Seguimos un proceso cíclico de construcción de la taxonomía. Por un lado, revisamos la forma en que algunas revistas de investigación, memorias de congresos y bases de datos nacionales e internacionales asignan términos clave a sus documentos, con dos propósitos centrales: (a) asegurarnos de que los términos clave que consideraban tenían su equivalente en la taxonomía de Funes y (b) asegurarnos que los documentos de esas fuentes podían ser clasificados apropiadamente en nuestra taxonomía. Por otro lado, varios expertos internacionales revisaron la taxonomía e intentaron clasificar sus propios documentos con ella. En este proceso cíclico modificamos varias veces su estructura y contenido.

En la figura 1 presentamos parte de la taxonomía de Funes correspondiente a matemáticas escolares, llegando al detalle de los términos clave que forman parte de estructuras numéricas, correspondiente a números. La taxonomía completa se encuentra en Gómez y Cañadas (2010).

Figura 1. Parte de la taxonomía de términos clave en Funes

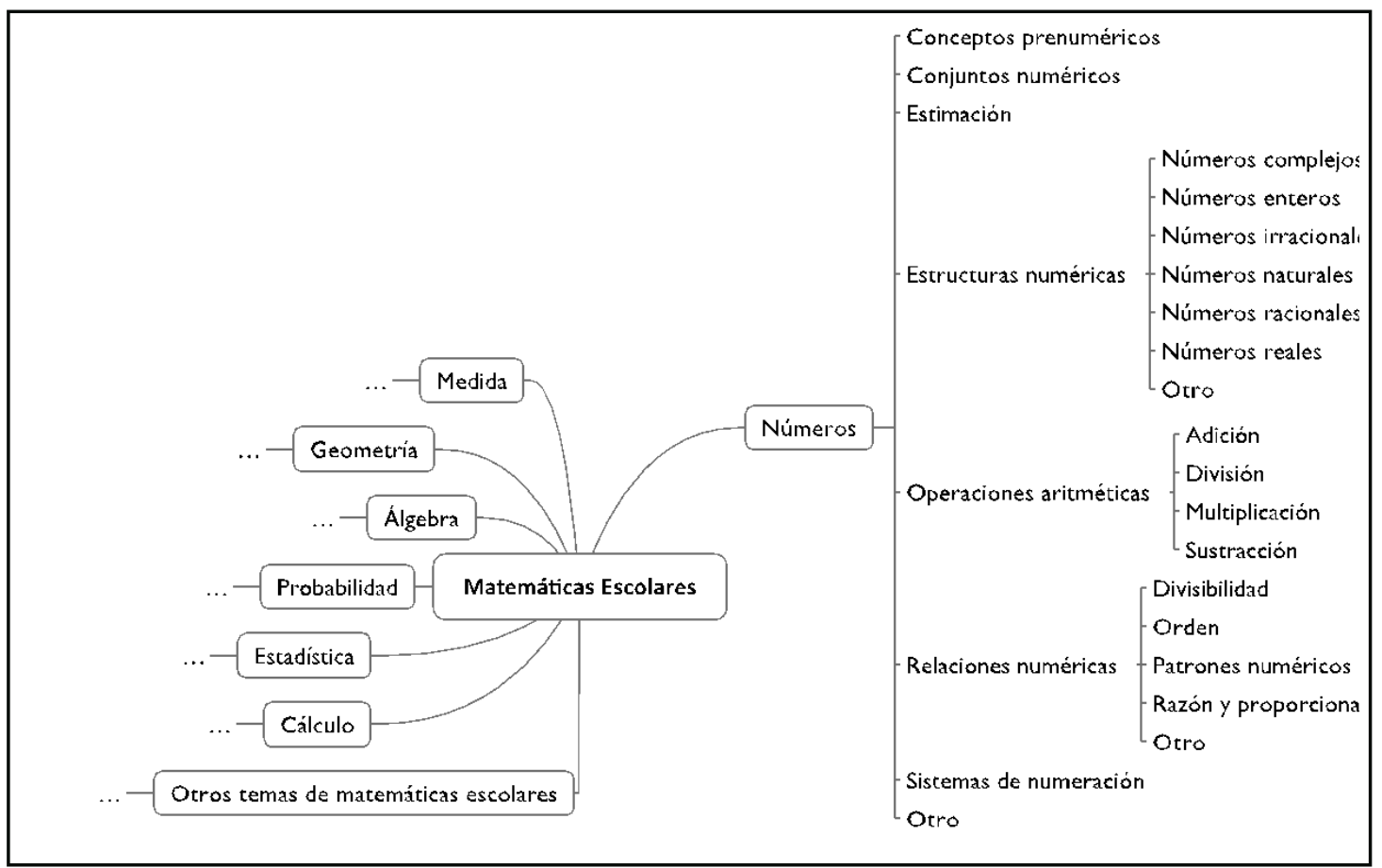

Fuente: Elaboración propia 
Precisión, relevancia y eficiencia de la taxonomía de Funes

Los problemas de ambigüedad se resuelven en Funes con base en su propia estructura. Por ejemplo, el término evaluación aparece en varios términos clave, pero estos términos clave se encuentran dentro de apartados que le dan el significado concreto que les corresponde. Abordamos la sinonimia de una manera indirecta. Cada concepto tiene un único término clave asignado en Funes. No obstante, el comité editorial mantiene una base de datos de sinónimos como medio para asegurar la completitud de la taxonomía.

Funes resuelve los problemas de relevancia y eficiencia a través del siguiente procedimiento. El usuario tiene acceso a la estructura de los términos clave con los que se ha etiquetado, al menos, un documento. El usuario puede pulsar en cualquiera de los términos clave para obtener la lista de documentos etiquetados con el término clave correspondiente. Al pulsar en el enlace que corresponde a un documento, el usuario obtiene la información detallada de ese documento y puede descargar el archivo correspondiente.

\section{Discusión}

En el contexto actual, el aprendizaje es un proceso social en el que aprendemos de manera interdependiente dentro de las múltiples comunidades de práctica a las que pertenecemos. La comunidad de Educación Matemática se configura alrededor de una variedad de personas (profesores, innovadores, investigadores, directivos, entre otros) que se agrupan en instituciones educativas, asociaciones y otros colectivos. Las tecnologías de la información y la comunicación presentan una oportunidad para que esta multiplicidad de profesionales se organicen y estructuren en un sistema social de aprendizaje a diferentes escalas, en el que cada quien pueda aprender de los demás y contribuir a su aprendizaje. Los repositorios digitales como Funes son una herramienta de este tipo de tecnología que puede contribuir a ese propósito.
Dadas las características de los tesauros y taxonomías existentes en la actualidad en Educación Matemática, en este documento hemos justificado la necesidad de construir una nueva taxonomía. Hemos presentado el marco conceptual en el que nos basamos para la construcción de la taxonomía de Funes y hemos indicado de qué forma esta taxonomía aborda los problemas de ambigüedad y sinonimia propios de los vocabularios controlados.

Consideramos que el procedimiento presentado para construir la taxonomía de términos clave de Funes se puede utilizar en otras disciplinas didácticas. La estructura curricular tiene carácter general. Por consiguiente, lo que se requiere es afinar los términos clave de la disciplina dentro de esa estructura curricular y establecer los contenidos de la disciplina tanto a nivel escolar, como avanzado.

\section{Agradecimientos}

Una versión extendida de este documento se publicó en Gómez y Cañadas (2013). Funes es un proyecto parcialmente subvencionado por el Ministerio de Educación Nacional de Colombia.

\section{Referencias}

ERIC. (2010). Mathematics education. Descargado el 5/9/2010, de http://www.eric.ed.gov:80/ ERICWebPortal/resources/html/thesaurus/ about_thesaurus.html

FIZ Kalruhe. (2010). MathEduc Database. Descargado el 5/9/2010, de http://www.zentralblatt-math.org/matheduc/classification/

Gómez, P. y Cañadas, M. C. (2010, 5/9/2010). Términos clave de Funes. Descargado el 5/9/2010, de http://funes.uniandes.edu. co/643/ 
Gómez , P. y Cañadas, M. C. (2013). Development of a taxonomy for key terms in mathematics education and its use in a digital repository. Library Philosophy and Practice (e-journal).

Gómez, P., Cañadas, M. C., Soler, M. y Restrepo, A. (2009). Funes: un repositorio digital para publicar y compartir documentos en Educación Matemática. En Sociedad Andaluza de Educación Matemática Thales (Ed.), Jornadas de investigación en el aula de matemáticas: dimensión histórica, social y cultural de las matemáticas (pp. 1-8). Granada: Editor.

Mullis, I. V. S., Martin, M. O., Ruddock, G. J., O'Sullivan, C. Y., Arora, A. \& Eberber, E. (2005). TIMSS 2007 assessment frameworks. Chestnut Hill, PA: Boston College.

NISO. (2005). Guidelines for the construction, format, and management of monolingual controlled vocabularies. Bethesda, MD: Autor.
Pinto, M. (2008). A user view of the factors affecting quality of thesauri in social science databases. Library \& Information Science Research, 30(3), 216-221.

Rico, L. (Ed.). (1997). Bases teóricas del currículo de matemáticas en educación secundaria. Madrid: Síntesis.

Tatto, T., Schwille, J., Schmidt, W., Ingvarson, L. \& Beavis, A. (2006). IEA teacher education study in mathematics (TEDS-M). Conceptual framework. East Lansing, MI: Teacher Education and Development International Study Center, College of Education, Michigan State University.

UNESCO. (2010). UNESCO thesaurus: alphabetical list. Descargado el 5/9/2010, de http:// www2.ulcc.ac.uk/unesco/terms/list93.htm - Mathematics\%20education 